\title{
Factors affecting implementation of perinatal mental health screening in women of refugee background
}

\author{
Nishani Nithianandan' ${ }^{1}$, Melanie Gibson-Helm¹, Jacquie McBride², Amanda Binny ${ }^{1}$, Kylie M. Gray ${ }^{3}$, \\ Christine East ${ }^{4,5}$ and Jacqueline A. Boyle 1* $^{*}$
}

\begin{abstract}
Background: For women of refugee background, the increased risk of mental illness associated with pregnancy is compounded by pre- and post-settlement stressors. In Australia, antenatal screening for depression and anxiety symptoms using the Edinburgh Postnatal Depression Scale is recommended for all women. Despite this, screening is not routinely implemented and little is known about barriers and enablers to implementation for women of refugee background.

Methods: Semi-structured interviews were conducted with a range of health professionals ( $n=28$ : midwives, obstetricians, perinatal mental health and refugee health experts, interpreters) and women of refugee background $(n=9)$. Themes generated from thematic analysis were examined in relation to the Theoretical Domains Framework and Cultural Competence Conceptual Framework, followed by identification of effective behaviour change techniques to address the barriers and enablers identified by participants. These techniques formed the basis of recommendations to inform sustainable implementation of screening and referral.
\end{abstract}

Results: Almost all participants perceived perinatal mental health screening to be necessary and most recognised the importance of post-traumatic stress disorder (PTSD) screening. Barriers and enablers were identified and related to eight domains: knowledge, skills, professional roles, beliefs about capabilities and consequences, environmental context, social influences and behavioural regulation.

Conclusions: This research clarifies how mental health screening may be integrated into routine antenatal care for women of refugee background, in order to improve provision of recommended care. These theory-informed recommendations include an inter-disciplinary approach, coordinating care within and across services, addition of PTSD screening, and effective communication with women.

Keywords: Refugees, Pregnancy, Prenatal care, Mental health, Depression, Anxiety, Post-traumatic stress disorder, Perinatal, Health services research

\section{Background}

Perinatal mental illness is a significant global problem, with postnatal depression prevalence ranging from 13 to $20 \%[1,2]$. Of women with postnatal depression, almost $40 \%$ are estimated to have developed symptoms during pregnancy [3]. Perinatal anxiety disorders may be as

\footnotetext{
* Correspondence: jacqueline.boyle@monash.edu

${ }^{1}$ Monash Centre for Health Research and Implementation, School of Public Health and Preventive Medicine, Monash University, 43-51 Kanooka Grove, Clayton 3168, Victoria, Australia

Full list of author information is available at the end of the article
}

prevalent as depression [4], and higher levels of antenatal anxiety increase the risk of postnatal depression [5].

Pregnancy is a time of heightened vulnerability for the development or recurrence of mental illness [6] and women of refugee background are likely to be at greater risk than the general population given pre- and postsettlement stressors [6, 7]. Estimates of mental illness prevalence in general refugee populations vary, with systematic reviews reporting depression prevalence of $5-31 \%$ and post-traumatic stress disorder (PTSD) 9-31\%, with evidence of significant 
comorbidity $[8,9]$. The prevalence of perinatal mental illness in this demographic is poorly documented. Throughout this paper, the term 'refugee background' refers to women who self-report either a refugee or asylum seeker background. Refugees are persons with a well-founded fear of persecution, who are outside of their country of origin and unable or unwilling to return, while asylum seekers are persons seeking protection whose refugee status is unconfirmed [10].

Perinatal mental illness presents a major public health challenge, given its contribution to maternal morbidity and indirect mortality [6], adverse obstetric outcomes [11], and impaired psychological and physical development of infants and children [12,13]. Partners' quality of life and mental health may also be affected, and other children in the family may experience a greater risk of mental illness and adverse social and behavioural outcomes $[6,14,15]$. Thus, there is a clear rationale for antenatal screening to identify early symptoms and provide appropriate follow-up and management to prevent exacerbation of symptoms and improve outcomes. Moreover, the regular contact between health professionals (HPs) and women during pregnancy supports the rationale for integrating screening into routine antenatal care [16].

Australian clinical practice guidelines recommend routine antenatal assessment of (i) psychosocial risk factors and (ii) depression and anxiety symptoms using the Edinburgh Postnatal Depression Scale (EPDS), an extensively used and validated perinatal screening tool $[6,17]$.

However, antenatal screening is not routinely implemented at many hospitals [18], and little is known about how to integrate mental health screening into antenatal care. Barriers include lack of time, funding or follow-up infrastructure and inadequate training $[6,19]$. Few enablers have been identified but include raising awareness amongst HPs, support from hospital management and development of follow-up pathways [19, 20].

Implementation is likely to be more complex for women of refugee background given their vulnerability and barriers to accessing health services such as lack of interpreters or healthcare literacy, Western medical models and stigma associated with mental illness [7, 21-23]. Previous studies of maternity care models with women of refugee background have not explored mental health screening $[21,22]$, system challenges or factors critical to success in rolling out a comprehensive screening and referral programme $[24,25]$.

Monash Health is located in south-east Melbourne in the Australian state of Victoria. It is one of the largest maternity service providers in Australia and also services a region with one of the largest resettled refugee populations in the country, up to $8.7 \%$ of the regional population $[10,26]$.
Importantly, a large proportion-40\% over the last 10 years-of persons resettled under Australia's Humanitarian Programme were women of child-bearing age [27]. Women self-reporting a refugee background are preferentially allocated to the Monash Health refugee antenatal clinic where possible. Psychosocial risk factor assessment, which aims to identify risk factors associated with perinatal mental illness such as past history of mental illness, current or past abuse, substance abuse and lack of social support [6], is routinely undertaken at Monash Health. However, screening for anxiety and depression symptoms is not undertaken at all, which is likely to lead to considerable under-recognition of women at risk of perinatal mental illness. This evidence-practice gap in antenatal care is widespread, with under a third of state public maternity hospitals reporting use of a psychosocial risk factor assessment tool and a quarter of hospitals reporting use of the EPDS [18]. This study aimed to (i) investigate barriers and enablers to implementing evidence-based, nationally recommended perinatal mental health screening and (ii) inform sustainable implementation of a screening and referral programme, in women of refugee background.

\section{Methods}

\section{Study design}

Qualitative research methods were deemed most appropriate to elicit in-depth stakeholder perspectives [28]. Semi-structured interviews were selected as they provide some guidance, while allowing the interviewer to be responsive to participants, empowering stakeholders to explore issues they identify as significant and providing an environment conducive to working with interpreters $[29,30]$. This decision was further supported as many interviewed women described their own struggles with mental illness, which may not have been volunteered in a group setting [29]. While in-person interviews afford greater opportunity to build rapport and observe body language, telephone interviews can be more convenient and less intimidating [29]. Hence, participants were offered both options.

\section{Materials}

Preliminary consultations with stakeholders suggested three areas of focus for the interview guide: (i) rationale for screening, (ii) acceptability of the screening tool and (iii) barriers and enablers to implementation of screening and referral. Based on these consultations, the interview guide (Appendix) was developed by three of the authors and pilot-tested with two independent researchers for feedback regarding open-ended enquiry, appropriate use of probes, and interview length. Once interviews with participants commenced, an iterative approach was adopted in which the interview guide was revised based on key ideas raised in previous interviews. For example, 
PTSD screening was discussed by one participant and then incorporated into the interview guide for subsequent interviews. The first named author and each participant jointly determined to what extent topics were explored, depending on the interviewee's role. The EPDS was brought as a prompt for community representative (CR) interviews.

\section{Recruitment}

Participants were approached during April-July 2015. HPs and researchers with specialist knowledge in perinatal mental health, refugee health or both, or who were involved in the Monash Health refugee antenatal clinic were purposively recruited. A broad range of stakeholders was sought because the topic intersects three distinct areas of healthcare: refugee health, pregnancy care and mental health and given the multidisciplinary nature of pregnancy care. All CRs and other HPs were recruited through snowballing techniques, which is useful in accessing 'hard-to-reach' populations [30]. CRs with a current or previous pregnancy and a refugee or asylum seeker background were recruited. Recruitment of CRs was assisted by a bicultural worker and Adult Multicultural Education Services volunteers, who introduced the study to potential participants and gauged interest before recommending them to the first named author. Accredited interpreters were arranged for nonEnglish-speaking CRs. Recruitment continued until data saturation was achieved.

\section{Participant characteristics}

The study sample included $28 \mathrm{HPs} /$ interpreters and 9 CRs from diverse ethnic backgrounds (Tables 1 and 2). Most individuals who were approached consented to participate in interviews (37/42). Two obstetricians failed to respond to interview requests, and three CRs were lost to contact. Of the CRs, four required interpreters.

\section{Data collection and analysis}

All interviews were audio-taped and transcribed verbatim by a professional transcription service. Transcripts were verified by the first named author, and participants were invited to review their transcripts. De-identified transcripts were imported into NVivo (Version 10) and thematic and inductive ('data-driven') analysis was conducted, involving data coding, reviewing the list of codes, organising codes within themes, revising themes against codes for inclusiveness and ongoing refinement of the thematic map [29]. Thematic analysis was conducted prior to identifying best fit theoretical framework(s) from the literature to avoid coding data to fit into a pre-existing framework [29]. Analysis was undertaken independently by two of the authors, with constructed themes compared for inconsistencies. This,
Table 1 Number of participants according to role

\begin{tabular}{|c|c|c|}
\hline Role & & $\begin{array}{l}\text { Number of participants } \\
(n=37)\end{array}$ \\
\hline \multirow[t]{12}{*}{ Staff } & Midwives & 5 \\
\hline & Obstetricians & 6 \\
\hline & $\begin{array}{l}\text { Maternal and child } \\
\text { health nurses }\end{array}$ & 2 \\
\hline & $\begin{array}{l}\text { Perinatal mental } \\
\text { health nurses }\end{array}$ & 2 \\
\hline & $\begin{array}{l}\text { Perinatal and infant } \\
\text { psychiatrist }\end{array}$ & 1 \\
\hline & $\begin{array}{l}\text { Perinatal mental } \\
\text { health expert }\end{array}$ & 1 \\
\hline & $\begin{array}{l}\text { Maternity general } \\
\text { practice liaison officer }\end{array}$ & 1 \\
\hline & $\begin{array}{l}\text { Community mental } \\
\text { health team leader }\end{array}$ & 1 \\
\hline & Refugee health nurse ${ }^{a}$ & 1 \\
\hline & Refugee health experts ${ }^{b}$ & 3 \\
\hline & Bicultural worker & 1 \\
\hline & Interpreters & 4 \\
\hline $\begin{array}{l}\text { Community } \\
\text { representatives }\end{array}$ & & 9 \\
\hline
\end{tabular}

Many participants occupied multiple roles; the most relevant role is listed ${ }^{a} \mathrm{~A}$ nurse or midwife trained to assess, educate, refer and coordinate care for people of refugee or asylum seeker background

${ }^{\mathrm{b}}$ Someone with extensive research or clinical experience with women of refugee background

along with researcher reflexivity, documentation of field notes and key analytic decisions and inclusion of representative quotes, contributed to research credibility, dependability and confirmability and thus analytic rigour [28].

Thematic analysis yielded a set of themes, which were closely examined in relation to the Theoretical Domains Framework (TDF) [31] and domain constructs to better understand behavioural determinants. The two analysts independently determined key domains to be those that described the barriers and enablers within the themes at a theoretical level. Interpretation of data was enhanced by the Cultural Competence Conceptual Framework

Table 2 Ethnic backgrounds of community representatives, interpreters and bicultural worker

\begin{tabular}{ll}
\hline Ethnic background & Number of participants $(n=14)$ \\
\hline Burmese (Rohingya) & 2 \\
Afghan & 5 \\
Sudanese & 1 \\
Iranian & 3 \\
Iraqi & 1 \\
Sri Lankan (Tamil) & 1 \\
Indian & 1 \\
\hline
\end{tabular}


(CCCF), which adds a cultural competence lens to the process of understanding behaviours and intervention design using the TDF. The CCCF describes three stages to achieving cultural competence: critical awareness and cultural knowledge, skills development and organisational support for multicultural practice [32]. As cultural competence was expected to be a key component of an appropriate screening programme in women of refugee background, the CCCF was deemed to provide an essential perspective not addressed by the TDF.

Of relevance to introducing a screening and referral programme, theory-informed, evidence-based, healthcare interventions are more effective than those lacking a theoretical foundation [31, 33, 34]. Both the TDF and CCCF are well-validated, successfully applied frameworks that encompass individual and organisational influences. Previous implementation studies have been informed by the mapping of TDF domains onto behaviour change techniques at an individual or organisational level [33-35] or have used two theoretical frameworks simultaneously to plan an optimal intervention [36].

In developing recommendations for a screening programme (Table 3), our understanding of what behaviours to target and how to effectively do so was informed by Michie et al.'s matrix, which maps theoretical domains (i.e. behavioural determinants) to effective behaviour change techniques using an expert consensus process [34]. After identifying potential behaviour change techniques for each domain, the research team and other members of a broader steering committee within the Monash Women's Maternity and Monash Health Refugee Health and Wellbeing services used their multidisciplinary experience to select the techniques most relevant to the setting. The same group developed recommendations (Table 3, columns 3 and 4) by applying these techniques to specifically address the barriers and enhance the enablers identified by participants.

\section{Results \\ Key findings}

Key findings are presented within eight key TDF domains (Fig. 1), which described the barriers and enablers within the themes at a theoretical level. The remaining six domains of the TDF were not relevant to the barriers and enablers.

\section{Knowledge}

Almost all participants perceived the need for, and understood the rationale behind, routine antenatal mental health screening:

...it is very very necessary because it's not only affects the woman herself but the whole family... I know it takes time and it needs money...but in order to make a better community we need to look into people's problems more deeply. Especially women because they are the core of the family...if they fall, everybody falls. Children, and husbands and partners you know everybody will be affected. (ID 4; interpreter)

One participant was ambivalent, citing the rigidity of universal screening, false positives and negatives, and inappropriate management. Few HPs volunteered knowledge of clinical practice guidelines regarding antenatal screening, and knowledge and experience of EPDS administration was variable.

While HPs commonly believed women of refugee background had a 'lack of understanding of mental health' (ID 24; HP), most CRs demonstrated some knowledge of mental health often drawn from their experience of symptoms (articulated as worry, crying, difficulty focusing, self-blame, 'pressure on brain', 'going mental', feeling depressed or sad) or mental health services (MHS). Many CRs and some HPs spoke about mental health in a broader psychosocial context. One interpreter insisted that mental illness was common but perinatal mental illness rare amongst Burmese women. Many HPs and CRs recommended community mental health education.

Multiple HPs explored the cumulative impacts of trauma, pregnancy and post-settlement stressors, and both HPs and CRs anticipated high prevalence of mental illness. Most women from culturally and linguistically diverse backgrounds described previous trauma impacting the foetus or leading to women not sleeping, eating or taking medication whereas HPs explored exacerbation of PTSD symptoms in pregnancy and its:

...significant impact on how women cope with pregnancy in terms of antenatal appointments... physical examinations... (ID 22; HP)

While most participants perceived PTSD screening as important, most lacked knowledge of trauma screening tools.

\section{Skills}

Most HPs advocated for staff 'training on not only how to screen but... what you're screening for and what does it mean and what do you do with that information?' (ID 9; HP) Some also recommended training in cultural competence and identification and prioritisation of refugee health needs:

...I don't think there's a lot of understanding with the midwives about, um, refugee health needs... the problem is they don't have a lot of background in this. (ID 27; HP) 


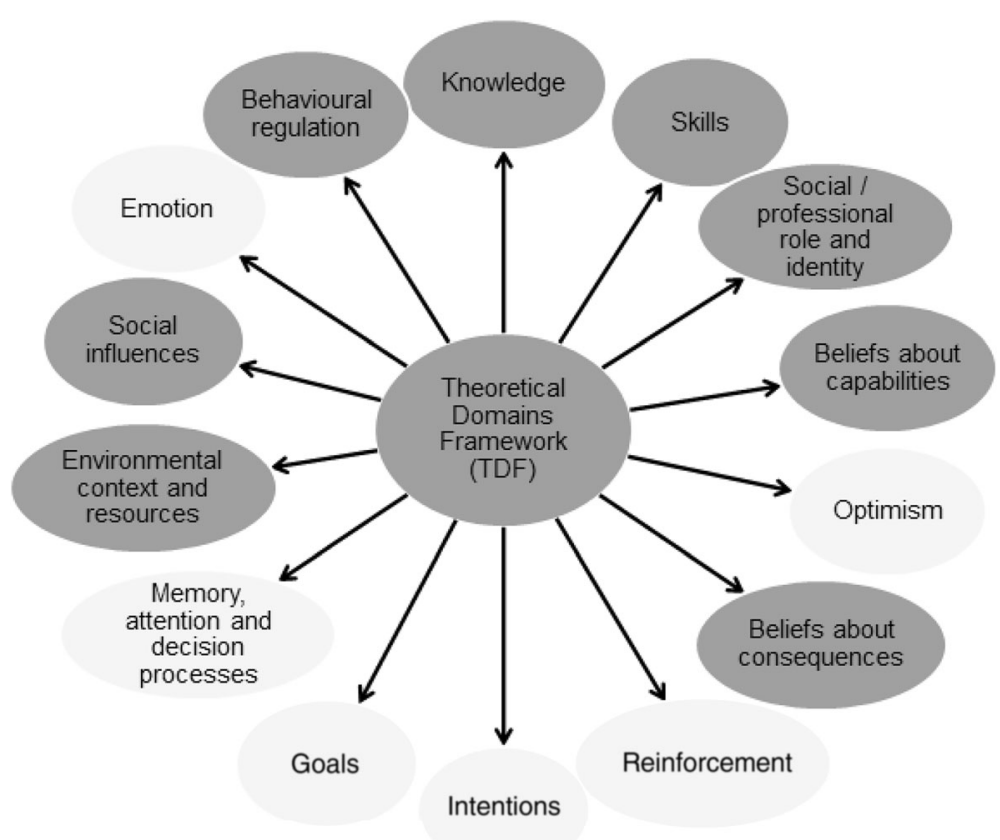

Fig. 1 Theoretical Domains Framework (dark grey circles represent domains relevant to key findings)

This perceived lack of understanding was thought to result in incomplete referrals, which failed to specify required services or the reason for referral beyond 'refugee background'.

\section{Social/professional role and identity}

All HPs asserted that they had a role to play in the implementation of screening, referral or management. Many described refugee health nurses acting as a 'go-to' person for staff and supporting women to access referrals:

.... face to face introduction to somebody in the Refugee Health Service such as [refugee health nurse] makes a huge difference to a woman's likelihood of accepting a referral to a service... (ID 10; HP)

Bicultural workers or community volunteers were widely perceived to improve cultural appropriateness of screening and follow-up. A few participants discussed the importance of perinatal mental health nurses in advising staff regarding women who are suicidal, at risk of self-harm or who have a significant history of mental illness and to facilitate appropriate triaging.

Midwives perceived their involvement throughout perinatal care but:

...our main focus would be identifying [cases] and referrals... (ID 14; HP)

Obstetricians advised that midwives administer screening-citing shorter obstetric appointments occurring later in pregnancy or the fact that midwives already undertook psychosocial risk factor assessment-though they outlined their responsibility to support referrals and monitor patients' progress.

A few HPs recommended involving social work and general practitioners. Participants from the Monash Health Refugee Health and Wellbeing service and community MHS felt that they had roles in providing referral pathways, triaging referrals and outreach support.

Participants desired clarity around roles within and between health services. A few HPs urged continuity and information sharing throughout the peripartum period to prevent:

...miscommunication and fragmentation to occur between GP [general practitioner], midwife, maybe an obstetrician if they get involved, Maternal and Child Health... and then all these other services; people fall through the gaps. (ID 25; HP)

\section{Beliefs about capabilities}

HPs expressed concern about when to refer, and who to refer to, and proposed that staff have access to telephone numbers and a support person onsite.

...a lot of times when I've had to deal with that it's been... a big learning curve at the time ... it's not streamlined and it's not efficient. It takes me a long time...to resource what I'm supposed to do about this kind of thing... (ID 14; HP) 
Some HPs suggested engaging staff in order to engage women to complete screening:

...if we're able to communicate the difference that this has the potential to make to women in their care they're far more likely to champion it...If we engage the staff they will engage the women. (ID 10; HP)

While HPs varied in their suggested approaches to PTSD screening, many urged a cautious, sensitive approach and acknowledged their lack of expertise:

I don't know how I would personally ask somebody if they'd experienced trauma. (ID 13; HP)

\section{Beliefs about consequences}

Both CRs and HPs identified stigma and apprehension around interpreters breaking confidentiality as potential barriers to women disclosing symptoms of mental illness and accessing MHS. Many CRs remarked that there may be differences between their own accepting attitudes towards mental illness and their communities' attitudes. A few CRs noted that their confidentiality concerns were allayed if they trusted the interpreter's professionalism.

CRs and interpreters generally perceived the EPDS as appropriate and easy to understand with an accurate interpreter:

...it's very appropriate to ask the refugee women like this kind of questions... (ID 36; CR)

Some HPs described the EPDS as straightforward and the best available screening tool; however, other HPs were unsure about its appropriateness, citing different cross-cultural understandings or manifestations of mental illness or noting that the EPDS overlooks trauma.

Normalising screening by 'talking about it...at previous appointments' (ID 8; HP), describing it as routine pregnancy care, sensitively framing MHS and providing follow-up care that women perceive to be useful to themselves or their children were identified as enablers to screening and referral uptake. Many CRs valued being able to express their feelings at counselling sessions ('I've emptied myself...' (ID 34; CR)) or desired provision of practical strategies, or both:

to be helpful...and more culturally suitable...it needs to give them some more practical advice...how to control their anxiety. (ID 4; interpreter)

Multiple CRs and HPs anticipated that the culturally competent, multidisciplinary service offered by the Monash Health Refugee Health and Wellbeing service would overcome many barriers to access.

\section{Environmental context and resources}

Access to accurate, on-site interpreters was unanimously discussed, with phone interpreters deemed inappropriate for mental health consultations. Several HPs perceived interpreters' skill as variable and a few suggested providing standardised instructions regarding EPDS translation. Many HPs and CRs advised having a female HP/ interpreter to improve cultural appropriateness, particularly for women with traumatic histories:

...with a female interpreter, we feel safe and we feel comfortable so everything that we wanted to express...we can say it easily... (ID 34; CR)

While many HPs identified the importance of translated screening tools, literal translation of the EPDS was widely considered inadequate and HPs advised back translation and gaining community input to better achieve cultural equivalence.

Most HPs identified lack of time as a barrier:

How much extra time do you need to allocate when you get .... high.... positive?...you need to have the capacity within your system to manage it if you've got someone who's suicidal... (ID 23; HP)

Participants were divided between screening as early as possible (e.g. most CRs) to facilitate earlier referral and waiting until a less time-pressured appointment when sufficient rapport had been built. Most obstetricians felt the first hospital visit with the midwife was sufficiently long; however, midwives insisted this visit was already very full. Some HPs suggested the second visit and advised repeating screening in third trimester or abstaining from screening if women were known to be linked-in with services:

...have some clinical discretion so that if you have a woman who presents with a really flat affect or is very teary or discloses spontaneously...that's the appropriate time to screen... (ID 10; HP)

Multiple HPs perceived screening as 'step one' (ID 24; $\mathrm{HP}$ ) and questioned the capacity and sustainability of MHS to accept referrals. A few urged initial consultation with and mapping of community MHS. Other common enablers included a private setting for screening (i.e. not the waiting room) and educating and assisting with transport.

\section{Social influences}

All CRs described loss of social support contributing to poor wellbeing. HPs and CRs considered family members to be protective against or contributors to mental 
illness or barriers to honest disclosure and help-seeking. A few HPs encouraged:

...making sure that the husband or other family members are okay with...the woman attending this type of referral... (ID 19; HP)

All CRs and some HPs regarded continuity of carers as critical to build trust, improve symptom monitoring and encourage disclosure:

...every day my doctor was changed I couldn't make a relationship with my...doctor. (ID 32; CR)

Several HPs prioritised having a 'go-to' person (i.e. refugee health nurse, senior staff, psychiatry liaison) for the staff who were administering screening. Infrequent suggestions included debrief opportunities, team meetings and support from hospital leadership.

\section{Behavioural regulation}

Factors that may facilitate changed practice at an individual or organisational level were identified by HPs. Some HPs identified the need for immediate follow-up for positive responses related to suicidal thoughts and long-term follow-up processes:

...the most important thing is follow-up... that if someone doesn't turn up for their appointment, that that's flagged...and that they're contacted. (ID 2; HP)

Most HPs discussed the need for clear referral guidelines (e.g. displayed as flowcharts in clinic rooms), and suggested that:

...those referral pathways are pretty simple if they're clearly articulated... (ID 28; HP)

Some HPs perceived that multiple referral pathways could be advantageous for different women's needs, whereas others anticipated that multiple pathways could be confusing for staff. Some participants anticipated improved referral uptake if follow-up care was provided on-site. Other critical success factors included minimising time between referral and follow-up, clear documentation, communication between services and feedback mechanisms to confirm receipt of referral.

\section{Discussion}

\section{Summary of key findings}

This study systematically defines barriers and enablers to implementation of perinatal mental health screening and referral for women of refugee background. Participants overwhelmingly recognised the need for mental health screening and PTSD screening. Factors affecting implementation identified by HPs included staff training needs, inter-disciplinary roles to support referral and clearly communicated, robust referral pathways. CRs prioritised continuity of care, female interpreters and HPs, social support and useful follow-up care. Key environmental considerations included availability of in-person interpreters, rigorously translated EPDS versions, time constraints and capacity of MHS.

Effective behaviour change techniques for each of the eight domains (i.e. behavioural determinants) were identified from Michie et al.'s matrix, which maps theoretical domains to behaviour change techniques. The multidisciplinary research team, including members from the Monash Women's Maternity and Monash Health Refugee Health and Wellbeing services, then selected the most relevant techniques to this setting, developed recommendations (Table 3) and compared these recommendations with the current literature.

\section{Context and implications for clinical practice Perceived need for perinatal mental health and PTSD screening}

The perceived necessity of perinatal mental health screening is an indicator of system readiness for change and is associated with a greater likelihood of effective implementation [37]. The perceived need to screen for PTSD symptoms in routine antenatal care of women of refugee background has not previously been reported, and specific PTSD screening may address the concern of a few HPs that the EPDS overlooks trauma. The varied opinions as to how to execute PTSD screening may reflect participants' concerns about exacerbation of symptoms if screening is inappropriately administered or differing success with screening methods. This highlights the need for careful selection of appropriate perinatal mental health and PTSD screening methods and training for HPs in sensitive administration (Beliefs about capabilities, Table 3).

\section{Inter-disciplinary approach}

The clear differences in roles attributed to each HP group and the recognised importance of refugee health nurse and perinatal mental health nurse roles uniquely highlight inter-professional relationships as important support structures for HPs involved in implementation [38] (Social/professional role and identity, Table 3). The perceived role of bicultural workers in educating and supporting women of refugee background is supported by the literature $[21,25]$ and by the CCCF, which asserts that individuals' awareness and knowledge alone are insufficient to result in culturally competent environments and organisations must also adapt services to be accessible to culturally and linguistically diverse patients [32]. 
Table 3 Recommendations for implementation of perinatal mental health screening in women of refugee background

\begin{tabular}{|c|c|c|}
\hline $\begin{array}{l}\text { Behavioural } \\
\text { determinant }\end{array}$ & Behavioural change techniques $^{\mathrm{a}}$ & Examples to support health professionals (HPs) \\
\hline Knowledge & $\begin{array}{l}\text { Information regarding } \\
\text { behaviour, outcome }\end{array}$ & $\begin{array}{l}\text { Provide information for HPs regarding } \\
\text { rationale for screening; clinical guidelines } \\
\text { and evidence-practice gap; appropriate } \\
\text { EPDS administration, scoring and actions; } \\
\text { and PTSD screening }\end{array}$ \\
\hline Skills & $\begin{array}{l}\text { Goal/target specified: } \\
\text { behaviour or outcome } \\
\text { Increasing skills: problem } \\
\text { solving, decision making, } \\
\text { goal setting } \\
\text { Rehearsal of relevant skills }\end{array}$ & $\begin{array}{l}\text { Organisation to set target of routine screening; } \\
\text { individual HPs to set targets for skills attainment } \\
\text { Provide training for HPs regarding } \\
\text { identification and prioritisation of refugee } \\
\text { health needs; appropriate use, scoring and } \\
\text { actions to EPDS; and cultural competence } \\
\text { (including approach to mental health and } \\
\text { managing family members) } \\
\text { Provide opportunities to practise skills }\end{array}$ \\
\hline
\end{tabular}

Social/professional Social processes of encouragement, Involve refugee health nurse, bicultural role and identity pressure, support worker, perinatal mental health nurse and senior staff to support referral Balance inter-disciplinary approach with clear delineation of roles Ensure clear communication between antenatal and postnatal services and identify women already receiving mental health care

Beliefs about capabilities

Beliefs about consequences

Environmental context and resources

Social influences

\section{Social processes of encouragement, pressure, support}

Environmental changes (e.g. objects to facilitate behaviour)

Persuasive communication Information regarding behaviour, outcome

Provide training for HPs (i.e. sensitive administration of trauma screening tool decision making, goal setting Social processes of encouragement, management of women at risk of suicide or self-harm) Engage staff by communicating the rationale for screening and benefits for women

Provide information for mental HPs regarding the provision of refugee appropriate mental health care (e.g. practical advice about managing symptoms)

Select the most appropriate time(s) to screen with input from HPs administering the EPDS (e.g. second antenatal visit and again in third trimester). Allow HPs discretion to screen earlier or later or to forgo screening if guided by MHS already involved in care Management to work with HPs to allow appropriate appointment length and flexibility to manage disclosures and make immediate referrals Map MHS in the area and confirm capacity and sustainability of services prior to implementation

Ensure a 'go-to' or support person for HPs (e.g. refugee health nurse, senior staff, psychiatry liaison), regular team meetings and debrief opportunities

Establish robust referral pathways, feedback mechanisms to confirm receipt of referrals, communication channels between services, and clear documentation at all stages of pathways Clearly communicate pathways (e.g.

flowcharts) and contact numbers for to HPs
Provide information (e.g. culturally appropriate group sessions, translated printed materials) at earlier appointments about perinatal mental illness, routine screening, and MHS

HPs to normalise screening; provide culturally appropriate mental health information at earlier appointments; manage expectations regarding referrals; and communicate professionalism of interpreters and usefulness of follow-up mental health care

Incorporate rigorously translated screening tools into routine maternity care Provide skilled, onsite, female interpreters for common refugee languages and standardised instructions for appropriate EPDS translation

Screen in a private setting

Provide advice around transport

Ensure continuity of care Include referral pathways to social work, women's groups and language services HPs to explain to family members what screening and potential follow-up involves; however screening to be undertaken privately

Establish various pathways for different needs while minimising referral points Use on-site services where possible (e.g. social worker) 
That all HPs asserted a role at one or more stages of screening, referral and management confirms the need for an inter-disciplinary approach in this setting.

Inter-disciplinary collaboration involves pursuing common goals, shared decision-making and planning and open communication between HPs and services [38] to ensure clarity around roles and appropriate, timely referrals and to prevent fragmentation of care. An example of shared planning is liaison with psychiatry departments and community MHS to assess their capacity to absorb referrals in the long-term (Environmental context and resources). Low rates of referral to specialist MHS have been found in the UK (1-3\% of women screened) and New South Wales, Australia, $[6,39]$ with women requiring less extensive care managed by general practitioners; however, referral rates may be higher for women of refugee background. Evaluation of local implementation efforts including referral rates would inform scaling-up of implementation. Shared decision-making is vital in determining optimal timing of screening administration, which is poorly covered in the literature. If midwives are to administer screening, recognising their perception of when it is practical to screen is likely to facilitate ownership and adoption of screening (Environmental context and resources, Table 3). In keeping with the 'organisational support' domain of the CCCF [32], health service managers need to work with HPs to allow flexibility to manage disclosures and make immediate referrals.

Inter-disciplinary collaboration also requires flexibility in sharing professional responsibilities to improve clinical care [38]. Not all HPs volunteered a direct role in screening. For instance, obstetricians recommended that midwives administer screening while they supported the referral and follow-up process; however, all HPs involved in antenatal care should receive training to appropriately fulfil their role whether they are directly involved in administering screening or not (Skills, Table 3).

\section{Effective communication with women}

Achieving cultural equivalence in EPDS translations and having accurate, female interpreters is consistent with the literature [21, 24, 40, 41]. Results from this study add that female interpreters are a priority specifically for mental health conversations to encourage disclosure from women with traumatic backgrounds. Translations of the EPDS are freely available for most refugee languages spoken in the local area [42]. Institutional investment, such as incorporating translated screening tools into routine maternity care and ensuring availability of onsite interpreters for common languages of resettled refugees, reflects the organisation's commitment to sustainable implementation [37] and is essential to a culturally competent service [32] (Environmental context and resources, Table 3 ).
Beliefs about consequences are known to affect implementation success [37] and persuasive communication and provision of information to HPs, and women are required to enhance and address these positive and negative beliefs respectively (Beliefs about consequences, Table 3). Some HP concerns regarding EPDS administration in this population are consistent with other research [24]. However, the positive perceptions of the EPDS by $\mathrm{CRs}$ and interpreters and their understanding of the impacts of perinatal mental illness contrast with a recent study, which described women's lack of understanding of EPDS items and concepts, and postulated that women of refugee background lacked a framework for understanding the rationale behind EPDS administration [24].

These differences may be attributed to greater exploration of CR understanding of the rationale for screening in this study; some CRs having experienced symptoms of mental illness or mental health care or differences in participant demographics (predominantly African compared with mostly Asian countries in this study), study design (focus groups and surveys compared with interviews) or familiarity with the EPDS. Further research is needed to investigate cross-cultural understandings of each EPDS item with women from key refugee communities.

Consistent with the literature $[23,40]$, there was some evidence that women of refugee background may use different words to articulate perinatal depressive symptoms or may misunderstand the 'perinatal onset' qualifier. Thus, when explaining the EPDS and undertaking diagnostic assessment, HPs need to consider women's different expressions for perinatal depression. However, many CRs also noted their own accepting attitudes towards mental illness differed from their communities', possibly stemming from personal experiences of mental illness and MHS, education or supportive family and friends. This challenges the notion of stigma and different cross-cultural understandings as insurmountable barriers. Along with CR understanding of the rationale, this supports the provision of culturally appropriate information about mental health and routine screening at earlier appointments to increase acceptance of later screening and follow-up (Knowledge and Beliefs about consequences, Table 3 ).

Normalising screening, managing expectations regarding referral(s) and communicating the professionalism of interpreters and usefulness of follow-up care are recommended to allay women's concerns and encourage engagement with services (Beliefs about consequences, Table 3). This study contributes to an under-researched area [43] and suggests that women of refugee background perceive 'useful' MHS to offer practical advice and opportunities to express emotions, thus providing valuable feedback for mental HPs. 


\section{Limitations and strengths}

While indistinct boundaries between some TDF domains may be considered a limitation, this reflects the often multiple determinants of behaviours and confirms that behaviour change requires a multi-faceted approach. A smaller number of CRs were recruited compared with HPs; however, data saturation was achieved to fulfil the study aims. While many of the CRs had accessed maternity services since coming to Australia (some of whom were currently pregnant) and these experiences informed their responses, this study did not specifically recruit CRs currently attending a maternity service. Current service users may identify additional barriers or enablers from their experience. To address this, recommendations from this study have informed a pilot programme designed by a broad steering committee and evaluation will include focus groups with women and health professionals. Strengths of this study include the range of participants interviewed, community consultation, rigorous study design and use of two generalizable, applied theoretical frameworks.

\section{Conclusions}

While clinical guidelines recommend integrating screening into routine antenatal care, this formative research is the first to clarify how integration might be achieved for women of refugee background. This study reveals a prevailing attitude amongst stakeholders that perinatal mental health and PTSD screening is necessary in this population. Findings emphasise the importance of adopting an inter-disciplinary approach to implementation and facilitating effective communication with women and between and within health services. This research provides clear evidence around barriers and enablers, and theory-based recommendations to inform implementation of perinatal mental health screening and referral for women of refugee background and ultimately improve provision of recommended antenatal care.

\section{Appendix}

\section{Intervention schedule}

1. Can you tell me about your work/yourself?

2. Have you had any experience with mental health screening programs or screening tools?

3. How necessary do you think mental health screening is?

4. How much do you know about the EPDS?

a. Do you think the EPDS is an appropriate questionnaire to assess mental health in this population?

5. What do you understand about the role of trauma in affecting pregnancy? a. What do you think about screening for trauma in pregnancy?

7. In your opinion, when during a woman's pregnancy is the most appropriate time to conduct mental health screening?

8. Can you think of any challenges to setting up screening at the antenatal clinic and its sustainability (i.e. factors that would undermine screening over the long-term)?

a. How might those challenges be overcome?

9. What factors might help screening to be as effective as possible?

10. How might we improve the cultural appropriateness of screening? (How can we make screening more culturally sensitive?)

11. Can you think of any challenges to the referral process?

12. What do you think critical success factors for a referral process would be?

13. What barriers might exist to women accepting follow-up care at the Refugee Health and Wellbeing Service? (Why might women not accept follow-up care at the Refugee Health and Wellbeing Service?)

14. How could the refugee antenatal clinic or the Refugee Health and Wellbeing Service support women to access follow-up care?

15. Do you see your organisation as having a role in the implementation of screening and referral, and if so what role?

16. Is there anything else you would like to add? a. Can you recommend anyone else we should speak to regarding implementation?

\section{Abbreviations}

CCCF: Cultural Competence Conceptual Framework; CR: Community representative; EPDS: Edinburgh Postnatal Depression Scale; HP: Health professional; MHS: Mental health services; PTSD: Post-traumatic stress disorder; TDF: Theoretical Domains Framework

Acknowledgements

The researchers wish to acknowledge the staff at the Monash Health Refugee Health and Wellbeing service and Monash Women's Clinic (Dandenong) for supporting this research, with particular thanks to Razia Ali, Maggie Lynch and Jackie McAsey for their assistance recruiting community representatives.

\section{Funding}

Parts of this project were supported by funding from Monash Health. MGH is supported by a National Health and Medical Research Council Early Career Fellowship (1110701)

Availability of data and materials

Data supporting findings may be accessed by contacting the corresponding author listed.

Author's contributions

Authorship credit should be based on (1) substantial contributions to

conception and design, acquisition of data, or analysis and interpretation of data; (2) drafting the article or revising it critically for important intellectual content and (3) final approval of the version to be published. Authors should 
meet conditions 1, 2 and 3. NN contributed to the study design, conducted the data acquisition, analysis and interpretation, drafted the manuscript and provides final approval of the version to be published. MGH led the study conception and design, supervised the data acquisition and analysis, contributed to the data interpretation, made critical revisions to the manuscript and provides final approval of the version to be published. JM made contributions to the study design and planning, contributed to the data acquisition, made critical revisions to the manuscript and provides final approval of the version to be published. AB conducted the data analysis and interpretation (independently of the first named author), made critical revisions to the manuscript and provides final approval of the version to be published. KMG made contributions to the study design, made critical revisions to the manuscript and provides final approval of the version to be published. CE made contributions to the study design, made critical revisions to the manuscript and provides final approval of the version to be published. JB led the study conception and project design, supervised the data acquisition and analysis, contributed to the data interpretation, made critical revisions to the manuscript and provides final approval of the version to be published.

\section{Competing interests}

The authors declare that they have no competing interests.

\section{Consent for publication}

Not applicable.

\section{Ethics approval and consent to participate}

Ethics approval was granted by the Monash Health Human Research Ethics Committee (project number 14475L). Verbal informed consent (taperecorded) was obtained for all interviews; written consent was additionally obtained for in-person interviews.

\section{Author details}

'Monash Centre for Health Research and Implementation, School of Public Health and Preventive Medicine, Monash University, 43-51 Kanooka Grove, Clayton 3168, Victoria, Australia. ${ }^{2}$ Monash Health Refugee Health and Wellbeing, Monash Health, Clayton, Victoria, Australia. ${ }^{3}$ Centre for Developmental Psychiatry and Psychology, Department of Psychiatry, School of Clinical Sciences, Monash University, Clayton, Victoria, Australia. ${ }^{4}$ Monash Women's Maternity Services, Monash Health, Clayton, Victoria, Australia. ${ }^{5}$ School of Nursing and Midwifery, Monash University, Clayton, Victoria, Australia.

Received: 23 May 2016 Accepted: 24 October 2016 Published online: 18 November 2016

\section{References}

1. O'Hara MW, Swain AM. Rates and risk of postpartum depression-a metaanalysis. Int Rev Psychiatry. 1996;8(1):37-54.

2. Fisher J, Mello MCD, Patel V, Rahman A, Tran T, Holton S, et al. Prevalence and determinants of common perinatal mental disorders in women in lowand lower-middle-income countries: a systematic review. Bull World Health Organ. 2012;90(2):139-49.

3. Green J, Murray D. The use of the Edinburgh Postnatal Depression Scale in research to explore the relationship between antenatal and postnatal dysphoria. London: Gaskell; 1994. p. 180-98.

4. Wenzel A, Haugen EN, Jackson LC, Robinson K. Prevalence of generalized anxiety at eight weeks postpartum. Arch Women's Ment Health. 2003;6(1):43-9.

5. Austin MP, Tully L, Parker G. Examining the relationship between antenatal anxiety and postnatal depression. J Affect Disord. 2007;101(1-3):169-74.

6. Austin MP, Highet N, The Guidelines Expert Advisory Committee. Clinical practice guidelines for depression and related disorders - anxiety, bipolar disorder and puerperal psychosis - in the perinatal period. A guideline for primary care health professionals. Melbourne: beyondblue: the national depression initiative; 2011.

7. Collins CH, Zimmerman C, Howard LM. Refugee, asylum seeker, immigrant women and postnatal depression: rates and risk factors. Arch Women's Ment Health. 2011;14(1):3-11.
8. Fazel M, Wheeler J, Danesh J. Prevalence of serious mental disorder in 7000 refugees resettled in western countries: a systematic review. Lancet. 2005;365(9467):1309-14.

9. Steel Z, Chey T, Silove D, Marnane C, Bryant RA, Van Ommeren M. Association of torture and other potentially traumatic events with mental health outcomes among populations exposed to mass conflict and displacement: a systematic review and meta-analysis. JAMA. 2009;302(5):537-49.

10. Department of Health, State of Victoria. The Victorian refugee and asylum seeker health action plan 2014-2018. Melbourne: State Government of Victoria; 2014.

11. Priest SR, Barnett B. Perinatal anxiety and depression: issues, outcomes and interventions. 2008.

12. Beck CT. The effects of postpartum depression on child development: a meta-analysis. Arch Psychiatr Nurs. 1998;12(1):12-20.

13. O'Connor TG, Heron J, Glover V, The ALSPAC Study Team. Antenatal anxiety predicts child behavioral/emotional problems independently of postnatal depression. J Am Acad Child Adolesc Psychiatry. 2002;41(12):1470-7.

14. Halligan SL, Murray L, Martins C, Cooper PJ. Maternal depression and psychiatric outcomes in adolescent offspring: a 13-year longitudinal study. J Affect Disord. 2007;97(1-3):145-54.

15. Murray L, Cooper PJ. The impact of postpartum depression on child development. Int Rev Psychiatry. 1996;8(1):55-63.

16. Yelland J, Brown SJ. Asking women about mental health and social adversity in pregnancy: results of an Australian population-based survey. Birth. 2014;41(1):79-87.

17. Australian Health Ministers' Advisory Council. Clinical Practice Guidelines: Antenatal Care - Module 1. Canberra: Australian Government Department of Health and Ageing; 2012.

18. Yelland J, Sutherland GA, Wiebe JL, Brown SJ. A national approach to perinatal mental health in Australia: exercising caution in the roll-out of a public health initiative. Med J Aust. 2009;191(5):276-9.

19. Buist A, Bilszta J. The beyondblue National Postnatal Screening Program, Prevention and Early Intervention 2001-2005, Final Report. Melbourne: beyondblue: the national depression initiative; 2006.

20. Darwin Z, McGowan L, Edozien LC. Antenatal mental health referrals: review of local clinical practice and pregnant women s experiences in England. Midwifery. 2015;31(3):e17-22.

21. Correa-Velez I, Ryan J. Developing a best practice model of refugee maternity care. Women Birth. 2012;25(1):13-22.

22. Stapleton H, Murphy R, Correa-Velez I, Steel M, Kildea S. Women from refugee backgrounds and their experiences of attending a specialist antenatal clinic. Narratives from an Australian setting. Women Birth. 2013;26(4):260-6.

23. Kokanovic R, Niner $S$, Cho V, Cuthbert D. Pilot study on perinatal depression (PD) in refugee communities. 2012. Research report prepared for beyondblue. Melbourne.

24. Stapleton $H_{1}$ Murphy $R$, Kildea S. Lost in translation: staff and interpreters' experiences of the edinburgh postnatal depression scale with women from refugee backgrounds. Issues Ment Health Nurs. 2013;34(9):648-57.

25. Johnson-Agbakwu CE, Allen J, Nizigiyimana JF, Ramirez G, Hollifield M. Mental health screening among newly arrived refugees seeking routine obstetric and gynecologic care. Psychol Serv. 2014;11(4):470-6.

26. Cheng I, Russell G, Bailes M, Block A. An evaluation of the primary healthcare needs of refugees in South East Metropolitan Melbourne. Melbourne: Southern Academic Primary Care Research Unit; 2011.

27. Settlement Reporting Facility [Internet]. Department of Immigration and Border Protection, Australian Government. [cited 16/05/2015]. Available from: http://www.immi.gov.au/settlement/srf/.

28. Liamputtong $P$. The science of words and the science of numbers: research methods as foundations for evidence-based practice in health. Research methods in health: foundations for evidence-based practice. 2010. p. 3-26.

29. Harding J. Qualitative data analysis from start to finish: Sage. 2013.

30. Liamputtong P. Performing qualitative cross-cultural research: Cambridge University Press. 2010

31. Cane J, O'Connor D, Michie S. Validation of the theoretical domains framework for use in behaviour change and implementation research. Implement Sci. 2012;7(1):37.

32. Balcazar FE, Suarez-Balcazar Y, Taylor-Ritzler T. Cultural competence: development of a conceptual framework. Disabil Rehabil. 2009;31 (14):1153-60.

33. French SD, Green SE, O'Connor DA, McKenzie JE, Francis JJ, Michie S, et al. Developing theory-informed behaviour change interventions to implement 
evidence into practice: a systematic approach using the Theoretical Domains Framework. Implementation Sci. 2012;7(1):38.

34. Michie S, Johnston M, Francis J, Hardeman W, Eccles M. From theory to intervention: mapping theoretically derived behavioural determinants to behaviour change techniques. Applied psychology. 2008;57(4):660-80.

35. Francis JJ, O'Connor D, Curran J. Theories of behaviour change synthesised into a set of theoretical groupings: introducing a thematic series on the theoretical domains framework. Implement Sci. 2012;7(1):35.

36. Tavender EJ, Bosch M, Gruen RL, Green SE, Michie S, Brennan SE, et al. Developing a targeted, theory-informed implementation intervention using two theoretical frameworks to address health professional and organisational factors: a case study to improve the management of mild traumatic brain injury in the emergency department. Implement Sci. 2015;10:74.

37. Greenhalgh T, Robert G, Macfarlane F, Bate P, Kyriakidou O. Diffusion of innovations in service organizations: systematic review and recommendations. Milbank Q. 2004;82(4):581-629.

38. D'Amour D, Ferrada-Videla M, San Martin Rodriguez L, Beaulieu M-D. The conceptual basis for interprofessional collaboration: core concepts and theoretical frameworks. J Interprof Care. 2005;19(S1):116-31.

39. Perinatal OM, Services MMH. Council Report CR88. London: Royal College of Psychiatrists; 2000

40. Laungani P. Postnatal depression across cultures: conceptual and methodological considerations. International Journal of Health Promotion and Education. 2000;38(3):86-94.

41. Kumar M, Ongeri L, Mathai M, Mbwayo A. Translation of EPDS questionnaire into Kiswahili: understanding the cross-cultural and translation issues in mental health research. J Pregnancy Child Health. 2015;2(1):134. doi: 10. 4172/2376-127X.1000134.

42. Department of Health, Government of Western Australia. Edinburgh Postnatal Depression Scale (EPDS): translated versions—validated. Perth: State Perinatal Mental Health Reference Group; 2006.

43. O'Mahony J, Donnelly T. Immigrant and refugee women's post-partum depression help-seeking experiences and access to care: a review and analysis of the literature. J Psychiatr Ment Health Nurs. 2010;17(10):917-28.

\section{Submit your next manuscript to BioMed Central and we will help you at every step:}

- We accept pre-submission inquiries

- Our selector tool helps you to find the most relevant journal

- We provide round the clock customer support

- Convenient online submission

- Thorough peer review

- Inclusion in PubMed and all major indexing services

- Maximum visibility for your research

Submit your manuscript at www.biomedcentral.com/submit 